\title{
Extraction of airway foreign bodies with bronchoscopy under general anesthesia in adults: an analysis of 38 cases
}

\author{
Yuling Wang, Juan Wang, Yinghua Pei, Xiaojian Qiu, Ting Wang, Min Xu, Jie Zhang \\ Department of Respiratory and Critical Care, Beijing Tian Tan Hospital, Capital Medical University, Beijing, China \\ Contributions: (I) Conception and design: Y Wang; (II) Administrative support: Y Wang, J Wang; (III) Provision of study materials or patients: Y \\ Wang, Y Pei; (IV) Collection and assembly of data: M Xu, J Zhang; (V) Data analysis and interpretation: X Qiu, T Wang; (VI) Manuscript writing: \\ All authors; (VII) Final approval of manuscript: All authors. \\ Correspondence to: Jie Zhang. Department of Respiratory and critical care, Beijing Tian Tan Hospital, Capital Medical University, 119 Nansihuan Xi \\ Lu, Fengtai District, Beijing 100070, China. Email: wylzxq0622@163.com.
}

\begin{abstract}
Background: To explore the efficacy and safety of extracting airway foreign bodies with bronchoscopy under general anesthesia in adults.

Methods: Altogether, 38 adult patients who underwent airway foreign body extraction with bronchoscopy under general anesthesia in the Pneumology Department of Beijing Tiantan Hospital, Capital Medical University, from January 2005 to December 2014 were included in the study and retrospectively analyzed concerning the extraction methods and complications. The indications and experience and lessons were summarized.

Results: In 38 patients with general anesthesia who had foreign bodies removed by bronchoscopy, 1 case failed to be removed, and 37 cases were successfully removed, with a success rate of $97.4 \%$. One of the patients failed to remove the foreign body due to massive hemorrhage during the resection of the right middle bronchial stone, and the operation was interrupted. Blood loss was analyzed in all patients, 4 patients $(10.5 \%)$ lost blood greater than $10 \mathrm{~mL}$, and 1 patient $(2.63 \%)$ lost blood greater than $300 \mathrm{~mL}$. To analyze the status of airway injury, 3 patients $(7.9 \%)$ had local airway mucosal tearing, and 1 patient had mild glottis edema. All the patients in this group had silent portal injury, severe hypoxemia or asphyxia, and no complications such as pneumothorax, mediastinal emphysema or pulmonary edema occurred.

Conclusions: Extraction of airway foreign bodies with bronchoscopy under general anesthesia in adults is safe and effective and can avoid surgical treatment in some patients.
\end{abstract}

Keywords: General anesthesia; bronchoscopy; adults; foreign airway body

Submitted Aug 24, 2020. Accepted for publication Oct 13, 2020.

doi: $10.21037 /$ jtd-20-2903

View this article at: http://dx.doi.org/10.21037/jtd-20-2903

\section{Introduction}

As early as 1897, German laryngologist g. Killian first used rigid bronchoscope to remove the pig bone from a male patient's trachea, thus avoiding tracheotomy. However, due to the limitation of equipment and operation technology, and the general anesthesia technology has not been developed, rigid bronchus needs to be carried out in conscious state, the patient's tolerance is poor, and skilled operators are required to use it, this technology has not been widely promoted. Of cases of foreign body aspiration into the airway, $80 \%$ occurs in children aged $<3$ years. In adults, it is less common and mostly occurs in patients with impaired consciousness or difficulty swallowing, e.g., patients with craniocerebral injury, intoxicated patients, and those under anesthesia $(1,2)$. Sometimes, foreign body aspiration may occur when coughing or laughing or during various accidents. Some patients are found to have foreign body retention because recurrent obstructive pneumonia resulted from accidental foreign body aspiration at a 
young age, which was not detected in time. Airway foreign bodies usually cause choking and complications, including recurrent cough, infection, and pulmonary atelectasis $(2,3)$ and can be life-threatening in severe cases. Small, flat, or round objects can be removed with flexible bronchoscopy under local anesthesia. However, if the foreign body is large, has a sharp edge, or has been kept for a long time or encapsulated by local tissue, it may not be easily removed through the glottis under local anesthesia and is more likely to cause airway injury and even asphyxia. If the patient cannot cooperate with physicians or tolerate the treatment, the procedure cannot be performed under local anesthesia; under such circumstances, the foreign airway body needs to be removed with flexible or rigid bronchoscopy under general anesthesia.

With the requirements of patients for the comfort of bronchoscopy, the development and wide application of general anesthesia, according to the needs of different operations, under general anesthesia through laryngeal mask, endotracheal intubation and rigid bronchoscopy to remove large, sharp and bleeding risk foreign bodies, which can avoid air duct injury, reduce complications, reduce the pain of patients, and improve the comfort of patients. And there are many instruments that can be used under rigid bronchoscope, and a variety of special forceps can be used to remove all kinds of foreign bodies, especially large foreign bodies. Therefore, it is the quickest and most reliable method to remove airway foreign bodies through rigid bronchoscope.

In this study, we have retrospectively analyzed the clinical data of 38 adults who had undergone extraction of airway foreign bodies with bronchoscopy under general anesthesia in the Pneumology Department of Beijing Tiantan Hospital, Capital Medical University, from January 2005 to December 2014.

We present the following article in accordance with the STROBE reporting checklist (available at http://dx.doi. org/10.21037/jtd-20-2903).

\section{Methods}

\section{General information}

Altogether, 38 patients (26 men and 12 women) were included in this study. Subjects' age ranged from 12 to 81 years, with an average age of 52.7 years. There were 30 cases of inhaled foreign bodies of exogenous origin, including metal or plastic pen caps, thumbtacks, dentures, teeth, various kinds of bones, nuts, kernels, and peppers. The duration of foreign body retention ranged from 1 day to 27 years, with an average duration of 37 months. There were 9 cases of accidental foreign body aspiration, accounting for $30.0 \%$, the largest proportion of all cases of endogenous foreign body aspiration. Eight patients had a history of central nervous system diseases, including cerebral infarction, cerebral hemorrhage, or coma after brain tumor surgery and thus had a sequela of coughing while eating $(26.7 \%)$. Five patients did not have a clear history of foreign body aspiration (16.7\%); 2 patients had accidental aspirations while sleeping after receiving sedatives $(6.7 \%)$. Two subjects had residual surgical sutures in their bodies $(6.7 \%)$. One patient had sudden aspiration at an extremely early age ( 2 years), and the foreign body was found after 27 years on physical examination. One patient's accidental aspiration was caused by a fall, which resulted from digestive tract hemorrhage and coma. In 1 patient, the clip for the occlusion of the tracheoesophageal fistula fell into the airway. Among the 30 patients abovementioned with endogenous foreign body aspiration, 17 had foreign bodies in their right bronchus, accounting for $56.7 \%$; 10 had foreign bodies in their left bronchus, accounting for $33.3 \%$; 2 patients had foreign bodies in both bronchi; the remaining one patient had a foreign body in the trachea. In 8 patients with endogenous foreign body aspiration, the foreign bodies were all bronchial stones of many sizes (see Table 1). This study was approved by the IRB of Beijing Tiantan Hospital Affiliated to Capital Medical University (No. JS2013-007-02). All procedures performed in this study involving human participants were in accordance with the Declaration of Helsinki (as revised in 2013). Individual consent for this retrospective analysis was waived.

Inclusion criteria: (I) patients who could not be taken out under local anesthesia, or patients could not tolerate and cooperate; (II) patients with large foreign body, sharp edge, difficult to pass through glottis under local anesthesia, and easy to damage the airway and even cause asphyxia; (III) patients with long stay time and serious local package, requiring high-frequency electrotome, laser, scissors and other high-risk operations; (IV) patients with risk of massive bleeding.

Exclusion criteria: (I) the patients with small foreign body and smooth edge, which are not easy to damage the airway or have the risk of asphyxia, can be removed under local anesthesia; (II) patients who can tolerate and cooperate under local anesthesia; (III) patients without obvious package around the foreign body, and do not need to use 
Table 1 Clinical features of 38 patients with airway foreign bodies

\begin{tabular}{lcc}
\hline Clinical feature & Number of cases & Percentage $(\%)$ \\
\hline Sex & 26 & 68.4 \\
Male & 12 & 31.6 \\
Female & 30 & 78.9 \\
Origin of the foreign body & 8 & 21.1 \\
Endogenous & & 2.6 \\
Exogenous & 1 & 60.5 \\
Location of the foreign body & 23 & 31.6 \\
Trachea & 12 & 5.3 \\
Right bronchus & 2 & \\
Left bronchus & & \\
Both bronchi & & \\
\hline
\end{tabular}

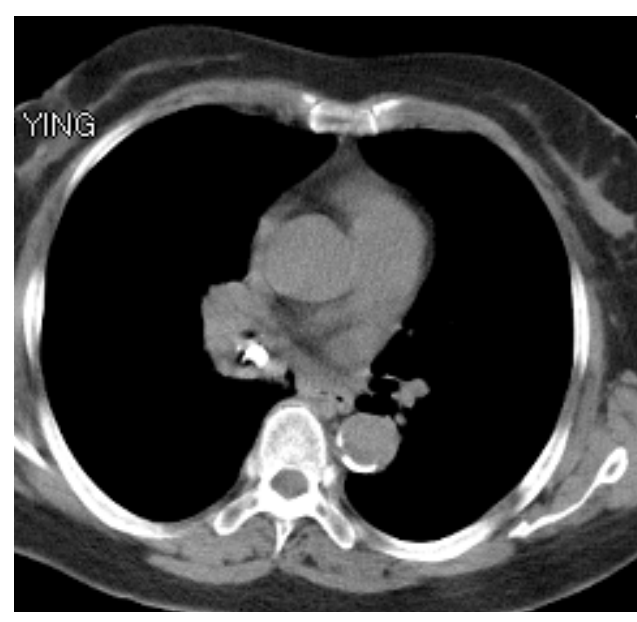

Figure 1 Bronchial stone in the right middle lobe.

high-frequency electric knife, laser, scissors and other highrisk operations; (IV) patients who cannot tolerate general anesthesia.

\section{Methods and complications}

All 38 patients underwent extraction of airway foreign bodies under general anesthesia. Among them, foreign bodies were extracted via flexible bronchoscopy in 14 patients (11 with laryngeal mask and 3 with tracheal intubation); rigid bronchoscopy was used in 24 patients, among which 12 patients underwent both rigid and flexible bronchoscopy. Thirty-seven foreign body extractions were

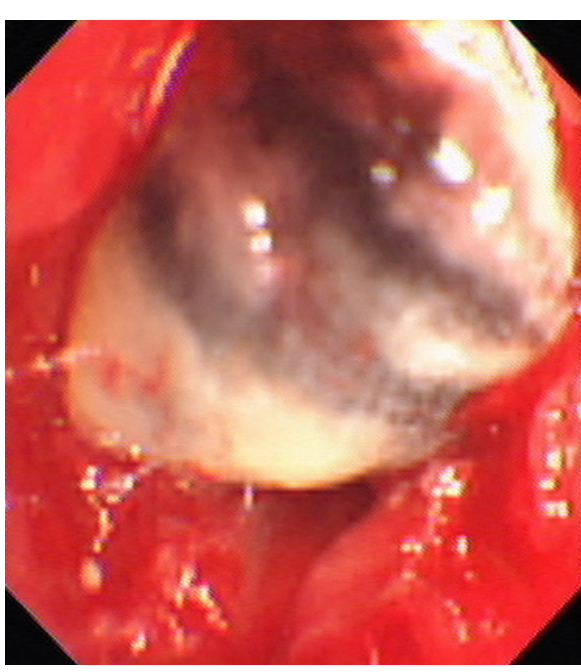

Figure 2 Bronchial stone in the right middle lobe.

successful, with a success rate of $97.4 \%$. One extraction failed because the operation was discontinued after massive hemorrhage during the process of extraction of bronchial stone in the right middle lobe bronchus (Figures 1,2). Of 38 patients, 4 had blood loss of $>10 \mathrm{~mL}(10.5 \%)$, among which one patient lost $300 \mathrm{~mL}$ of blood, three patients had local airway mucosal tears (7.9\%), and 1 had mild glottic edema, which was relieved after topical application of dexamethasone (Figures 3,4). No patient had glottic injuries, severe hypoxemia, or asphyxia. No patient had complications, including pneumothorax, mediastinal emphysema, and pulmonary edema (Table 2). 


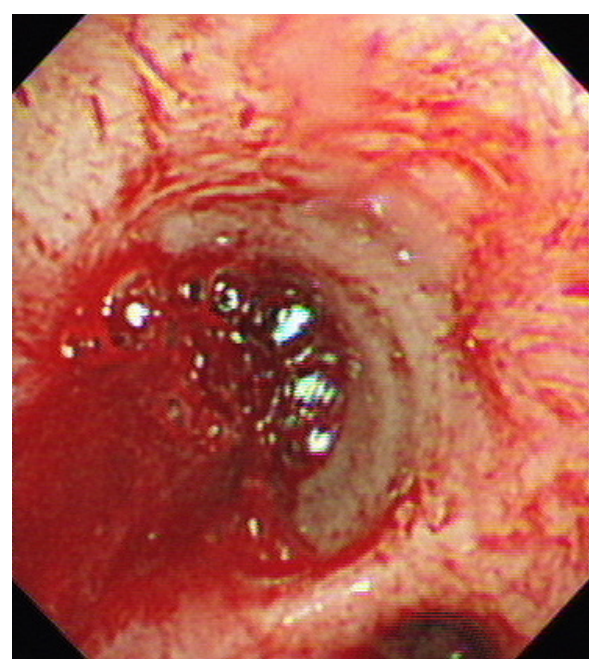

Figure 3 Hemorrhage during the extraction of bronchial stone.

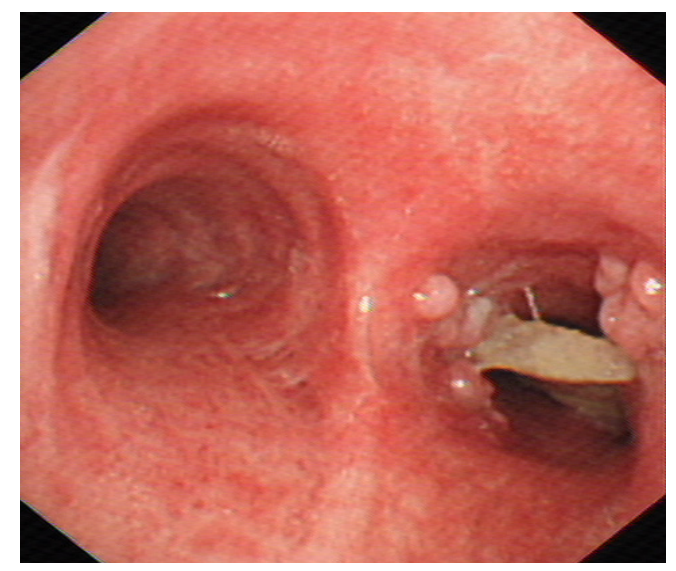

Figure 4 Foreign body in the right main bronchus: denture.

\section{Statistical methods}

SPSS 22.0 software was used for statistical analysis, and enumeration data are expressed as rate or constituent ratio.

\section{Discussion}

Airway foreign bodies can occur at any age and often expose the patients to the risk of pulmonary infections, pulmonary atelectasis, and even asphyxia. It is more commonly observed in children and less likely in adults (4). Patients with exogenous airway foreign bodies often have a history of accidental aspiration, and most of them can seek medical attention while they are conscious; they can receive timely treatment after undergoing imaging tests, including chest radiographs, CT, and bronchoscopy. However, in a patient with difficulty swallowing, with impaired consciousness, in an intoxicated state, or under anesthesia, the diagnosis often requires longer time, and even misdiagnosis may occur (3). A study showed that, in adults, nervous system diseases, head injuries, and intoxication are substantial risk factors for foreign body aspiration (5). Among the 38 patients with airway foreign bodies included in our study, eight patients had conditions, including cerebral infarction and cerebral hemorrhage. With difficulty swallowing, patients with such diseases are likely to have accidental aspirations while eating. With reduced coughing reflex, accidental aspiration is unlikely found as it occurs; it is often found after they seek medical care for recurrent pulmonary infection. Thus, patients are likely to miss prompt treatment and may have serious consequences, including pulmonary function impairment.

Table 2 Methods of extraction of foreign bodies and complications

\begin{tabular}{lcc}
\hline Project & Number of cases & Percentage (\%) \\
\hline Methods used to extract foreign bodies & & 26.3 \\
Rigid bronchoscopy & 10 & 36.8 \\
Flexible bronchoscopy & 14 & 36.8 \\
Both rigid and flexible bronchoscopy & & 10.5 \\
Complications & 4 & 7.9 \\
Hemorrhage & 3 & 2.6 \\
Airway mucosal tears & 1 & 78.9 \\
Glottic edema & 30 & \\
No complications & & \\
\hline
\end{tabular}


Several studies in China and other countries suggested that aging is a crucial factor for foreign body aspiration in adults $(6,7)$. With reduced epiglottic function or abnormal motor coordination, elderly patients are more likely to cough while eating, causing foreign bodies to enter the airway. In our study, there were 16 elderly patients aged $\geq 60$ years, accounting for $42.1 \%$ of the total number; among the elderly patients, 3 had bronchial stones, 1 had surgical suture in the bronchus, and the remaining 12 had foreign body aspiration. Therefore, if an elderly patient has a persistent cough, wheezing, dyspnea, or recurrent pulmonary infection in the same site, he/she is likely to have foreign body inspiration and needs related examinations to avoid delayed diagnosis. Concerning location, foreign bodies are more likely in the right bronchus for anatomical reasons; in this study, the foreign bodies were mainly in the right medial bronchus and right lower basal segmental bronchus, which is consistent with the reports in other studies.

In operation, we established the operating channel with a laryngeal mask, tracheal intubation, and rigid bronchoscopy and completed the extraction of foreign bodies from the trachea, right and left main bronchi, and segmental and subsegmental bronchi using the combination of flexible and rigid bronchoscopy, with a high success rate of $97.4 \%$. Our rate is like the success rate of $96.5 \%$ reported in a study where the extraction was completed using only flexible bronchoscopy (4), while, in our study, patients had less pain and glottic injuries were avoided. However, for airway foreign bodies that are large, have an irregular or sharp edge, or are likely to cause asphyxia, rigid bronchoscopy is undoubtedly the first choice of treatment (8). It can also be used in cases of airway foreign bodies found in the medial and lower segments of the bronchi and the bronchi below this level. Among the included cases, 24 extractions were completed using rigid bronchoscopy, accounting for $63.2 \%$ of all cases. Using this method has effectively protected the glottis, avoided mucosal damages and tears, and prevented foreign bodies from falling while being removed through the glottis, thus increasing the success rate of extraction. For foreign bodies that are relatively large and cannot pass the tube of the rigid bronchoscope, rigid forceps can be used to hold the foreign body and retract it along with the bronchoscope, which will reduce the risk of asphyxia and severe hypoxemia.

The success rate of removing foreign bodies under flexible bronchoscopy was $61-96.5 \%$, and the highest rate of removing foreign bodies by skilled doctors was $96.5 \%$, but there were still a few cases of failure. Under general anesthesia, the success rate of Flexible Bronchoscopy Combined with rigid bronchoscopy was about $97.4 \%$, but the pain of patients was less, and glottic injury was avoided. However, for large, irregular, sharp and easily asphyxiated airway foreign bodies, rigid mirror is undoubtedly the preferred method. In this paper, one case of failed removal of foreign body was a case of bronchial stone. The stone was partly in the bronchial lumen and partly in the bronchial wall. During the removal of the bronchial stone, the bleeding volume was $300 \mathrm{~mL}$, which led to the failure of removal. The patient was transferred to thoracic surgery for thoracotomy. Another patient inhaled sheep bone, and the broken sheep bone fell into the 4TH-5TH grade bronchus. He experienced a failure to remove foreign body from soft bronchus under local anesthesia, one failed to remove foreign body under laryngeal mask soft bronchoscope under general anesthesia, and the third time under general anesthesia and hard bronchoscope combined with soft bronchoscope, which avoided thoracic surgery. To sum up the above experience, (I) small and hard foreign bodies falling into the distal bronchus are easy to lead to removal failure; (II) part of the stones buried in the bronchial wall, especially most of them buried in the bronchial wall and closely related to the surrounding large blood vessels, may lead to the failure of removal. Therefore, it is necessary to improve the chest enhanced CT, read the film carefully and observe the results before operation. The position of the stone and its relationship with the surrounding tissues can reduce the complications during operation. (III) For thin and sharp foreign bodies, such as metal nails, long and thin bone bones, fishbone, blade, etc., if the foreign body penetrates into the tissues outside the bronchial wall, especially the large vessels in the mediastinum, it is not recommended to remove the foreign bodies under bronchoscope, and surgical treatment is recommended.

However, there are more advantages and instruments under rigid bronchoscope under general anesthesia. A variety of special forceps can be used to remove all kinds of foreign bodies, especially large foreign bodies. Therefore, it is the quickest and most reliable method to remove airway foreign bodies through rigid bronchoscope. And some foreign bodies cannot be taken out without hard mirror, such as large foreign body stuck in airway, sharp metal nail, slender bone, fish bone, etc. the metal sheath of hard mirror can effectively protect the throat, glottis and upper trachea, and facilitate the entry and exit of various instruments and sharp foreign bodies. The disadvantage 
is that open bronchoscopy under rigid bronchoscope is not stable enough, and the operation time is long, which leads to lack of $\mathrm{O}_{2}$ or $\mathrm{CO}_{2}$ retention. However, with the clinical application of new high frequency jet ventilator, this defect can be overcome. In addition, some patients with short mandible, head and neck, temporomandibular joint movement limitation, laryngeal stenosis and other patients may fail to insert hard mirror.

From our experience in foreign body extraction for 38 adults, the success rate of exogenous foreign body extraction is the highest at $100 \%$. However, in patients with foreign bodies that have remained for a long time, are encapsulated by hypertrophic granulation, or have unclear structure, extraction can be challenging and even require multiple surgeries to complete. For endogenous foreign bodies like bronchial stone, extraction is much more challenging, and its completion depends on the size and location of the object, whether it is deeply hidden in the bronchial wall and its relationship with surrounding blood vessels. In this study, 7 of 8 extractions of bronchial stones were successful, among which three patients had a blood loss of $>10 \mathrm{~mL}$ during the operation. In the unsuccessful case, the operation was discontinued after the massive hemorrhage $(300 \mathrm{~mL})$ during the extraction process, and the patient was then transferred to the Department of Surgery to perform foreign body extraction via thoracotomy. Therefore, we need to test this type of patient thoroughly and be prepared to ensure patient safety.

The most common complication of airway foreign body extraction is airway mucosal damage; sometimes, airway rupture may occur, leading to pneumothorax, mediastinal emphysema, and bronchial fistula and even life-threatening conditions in severe cases. In 38 included patients, 3 had local airway mucosal tears, resulting in different amounts of bleeding (blood loss was $20 \mathrm{~mL}$ in 1 patient), but none of the patients had complications including pneumothorax and mediastinal emphysema. Therefore, when performing extraction of airway foreign bodies under general anesthesia, we need to test the size of the foreign body and manipulate the direction of extraction carefully by placing the long axis of the foreign body parallel to the airway. Instead of violently pulling it out, the foreign body needs to be gently extracted to avoid serious complications.

Therefore, as the technology of interventional bronchoscopy develops, the techniques of airway foreign body extraction in adults have also improved. The combination of rigid and flexible bronchoscopy has significantly increased the success rate of foreign body extraction and reduced the development of complications. With this approach, many patients can avoid undergoing thoracotomy and thus have less pain and financial burden. Therefore, this method deserves further clinical application.

\section{Acknowledgments}

Funding: None.

\section{Footnote}

Reporting Checklist: The authors have completed the STROBE reporting checklist. Available at http://dx.doi. org/10.21037/jtd-20-2903

Data Sharing Statement: Available at http://dx.doi. org/10.21037/jtd-20-2903

Conflicts of Interest: All authors have completed the ICMJE uniform disclosure form (available at http://dx.doi. org/10.21037/jtd-20-2903). The authors have no conflicts of interest to declare.

Ethical Statement: The authors are accountable for all aspects of the work in ensuring that questions related to the accuracy or integrity of any part of the work are appropriately investigated and resolved. This study was approved by the IRB of Beijing Tiantan Hospital Affiliated to Capital Medical University (No. JS2013-007-02). All procedures performed in this study involving human participants were in accordance with the Declaration of Helsinki (as revised in 2013). Individual consent for this retrospective analysis was waived.

Open Access Statement: This is an Open Access article distributed in accordance with the Creative Commons Attribution-NonCommercial-NoDerivs 4.0 International License (CC BY-NC-ND 4.0), which permits the noncommercial replication and distribution of the article with the strict proviso that no changes or edits are made and the original work is properly cited (including links to both the formal publication through the relevant DOI and the license). See: https://creativecommons.org/licenses/by-nc-nd/4.0/.

\section{References}

1. Limper AH, Prakash UB. Tracheobronchial foreign bodies in adults. Ann Intern Med 1990;112:604-9. 
2. Chen CH, Lai CL, Tsai TT, et al. Foreign body aspiration into the lower airway in Chinese adults. Chest 1997;112:129-33.

3. Ng J, Kim S, Chang B, et al. Clinical features and treatment outcomes of airway foreign body aspiration in adults. J Thorac Dis 2019;11:1056-64.

4. Dong YC, Zhou GW, Bai C, et al. Removal of tracheobronchial foreign bodies in adults using a flexible bronchoscope: experience with 200 cases in China. Intern Med 2012;51:2515-9.

5. Mise K, Jurcev Savicevic A, Pavlov N, et al. Removal of tracheobronchial foreign bodies in adults using flexible

Cite this article as: Wang Y, Wang J, Pei Y, Qiu X, Wang T, Xu M, Zhang J. Extraction of airway foreign bodies with bronchoscopy under general anesthesia in adults: an analysis of 38 cases. J Thorac Dis 2020;12(10):6023-6029. doi: 10.21037/jtd20-2903 bronchoscopy: experience 1995-2006. Surg Endosc 2009;23:1360-4.

6. Liu Wj, Yang J, Zhu SY. A retrospective analysis of 22 cases of treating airway foreign bodies in elderly patients using flexible bronchoscopy. Guizhou Med J 2012;36:1092-4.

7. Ramos MB, Fernández-Villar A, Rivo JE, et al. Extraction of airway foreign bodies in adults: experience from 19872008. Interact Cardiovasc Thorac Surg 2009;9:402-5.

8. Rafanan AL, Mehta AC. Adult airway foreign body removal. What's new? Clin Chest Med 2001;22:319-30.

(English Language Editor: J. Chapnick) 